\title{
Excimer Laser Crystallization Processes of Amorphous Silicon Thin Films by Using Molecular-dynamics Simulations
}

\author{
Shinji MUNETOH, ${ }^{1)}$ Xiao Yan PING, ${ }^{1)}$ Tomohiko OGATA, ${ }^{1)}$ Teruaki MOTOOKA ${ }^{21}$ and Ryo TERANISHI ${ }^{11}$ \\ 1) Department of Materials Science and Engineering, Faculty of Engineering, Kyushu University, 744 Motooka, Nishi-ku, \\ Fukuoka 819-0395 Japan. $\quad$ 2) Kyushu University Cleanroom Laboratory Facility, Kyushu University, 8-7 Yayoigaoka, Tosu, \\ Saga 841-0005 Japan.
}

(Received on April 19, 2010; accepted on July 27, 2010)

\begin{abstract}
Crystallization processes of amorphous Si during the excimer laser annealing in the complete-melting and near-complete-melting conditions have been investigated by using molecular-dynamics simulations. The initial amorphous Si MD cell was prepared by quenching a liquid Si layer with 18666 atoms. KrF excimer laser annealing processes of amorphous Si were calculated by taking account of the change in the optical constant upon melting during a Gaussian-shape laser pulse shot with full width at half maximum (FWHM) of $25 \mathrm{~ns}$. The simulated results well reproduced the observed melting rate and the near-complete-melting and complete-melting conditions were obtained for 160 and $180 \mathrm{~mJ} / \mathrm{cm}^{2}$ fluence, respectively. It was found that larger grains were obtained in the near-complete-melting condition. Our MD simulations also suggest that the nucleation occur from unmelted amorphous Si region during laser irradiation and crystal growth proceeds toward supercooled $l$-Si region in the near-complete-melting condition.
\end{abstract}

KEY WORDS: molecular-dynamics simulation; excimer laser annealing; amorphous silicon.

\section{Introduction}

Excimer laser annealing is an effective technique in order to obtain polycrystalline silicon (poly-Si) deposited on glass substrate. Recently, various studies for "system-ondisplay" in which both active-matrix switches and control circuits are formed on glass have been performed. In order to improve the device performance, high-quality poly-Si thin films are required. In the excimer laser annealing, crystallization is occurred by melting of amorphous Si films deposited on glass and subsequent quenching of liquid Si ( $l$ $\mathrm{Si}$ ) using irradiation of an excimer laser pulse shot with a few $10 \mathrm{~ns}$ width. Hatano et al. previously reported the relationship between grain sizes and excimer laser fluences. ${ }^{1)}$ At low laser fluence, amorphous $\mathrm{Si}$ is partially melted and poly-Si composed of only small grain sizes is obtained. The grain size becomes larger as the laser fluence increases and the largest grain size of poly-Si can be obtained when the laser fluence melts almost all of the amorphous $\mathrm{Si}$ film, which is called "near-complete melting". On the other hand, the grain size decreases as the laser fluence further increases.

The molecular-dynamics (MD) simulation is a powerful tool for analyzing the crystallization processes in atomic scale. We previously reported the mechanism of nucleation in the various laser fluence by MD simulations. ${ }^{2-4)}$ The temperature range for the highest growth rate was found to be approximately 500 degrees higher than that for the highest nucleation rate. It was also found that a steady state temperature gradient was obtained in the direction of the surface normal during laser irradiation. In this paper, we have di- rectly observed crystallization processes of the excimer laser annealing in the complete and near-complete condition by using MD simulations.

\section{Calculation Method}

The initial MD cell was prepared by quenching of $l-\mathrm{Si}$ with a size of $50 \times 50 \times 150 \AA^{3}$ composed of 18666 atoms. MD simulation was performed by using Tersoff potential. ${ }^{5,6)}$ In order to compare with experimental data, the paircorrelation functions $g(r)$ for liquid $\mathrm{Si}$ at $3000 \mathrm{~K}$ and amorphous $\mathrm{Si}$ at $500 \mathrm{~K}$ were shown in Fig. 1. These functions were obtained by averaging over 200 configurations during

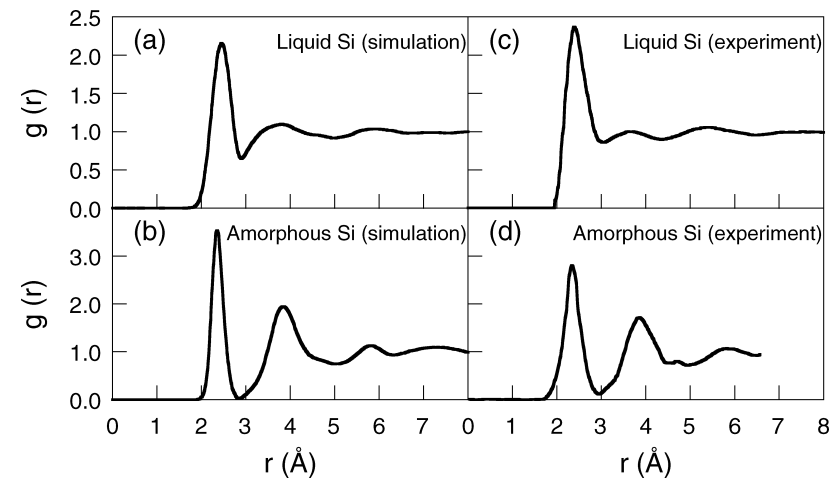

Fig. 1. Pair-correlation functions $g(r)$ for (a) liquid $\mathrm{Si}$ and (b) amorphous Si obtained from MD simulations using Tersoff potentials. The experimental data are also indicated, (c) liquid $\mathrm{Si}$ and (d) amorphous $\mathrm{Si}^{7,8)}$ The simulated temperatures of (a) and (b) are 3000 and $500 \mathrm{~K}$, respectively. 
200 MDstep (0.4 ps). The positions of peaks are in good agreement with the experimental ones ${ }^{7,8)}$ in both cases. In order to clarify the dynamical properties of amorphous $\mathrm{Si}$ generated by the Tersoff potential, the phonon densities of states were calculated from the Fourier transform of the velocity autocorrelation functions (shown in Fig. 2). The peaks around 20,40, and $60 \mathrm{meV}$ correspond to the acoustic and optical vibrational modes of c-Si. These positions are in good agreement with the experimental ones ${ }^{9)}$ indicated by open circles, suggesting that amorphous $\mathrm{Si}$ generated from the Tersoff liquid can reproduce not only the static structures but also dynamical properties of actual amorphous Si. Although by using Tersoff potential the melting points of crystalline and amorphous Si are approximately 2580 and $2000 \mathrm{~K}$, respectively, different to the experimental value, the structures of $l$-Si or amorphous Si can be well reproduced with this potential. Periodic boundary conditions were used in $X$ and $Y$ directions. In the $Z$ direction, atoms with the $Z$-coordinates less than $3 \AA$ were fixed, while the top surface atoms of the amorphous Si layer were set to be free. The whole MD cell was heated at $300 \mathrm{~K}$ for $50 \mathrm{ps}$ in order to relax structure with the time step of $2 \mathrm{fs}$. After structure relaxation of amorphous $\mathrm{Si}$, we performed MD simulations in order to reproduce melting and crystallization under the excimer laser irradiation. $\mathrm{KrF}$ excimer laser (wavelength: $248 \mathrm{~nm}$ ) was assumed a Gaussian-shape laser pulse with full width at half maximum (FWHM) of

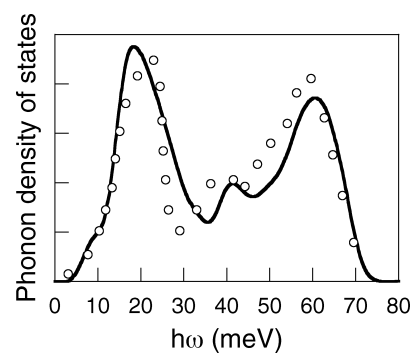

Fig. 2. Phonon density of states of amorphous Si obtained from MD simulation. Open circles indicate an experimental data. $^{9)}$

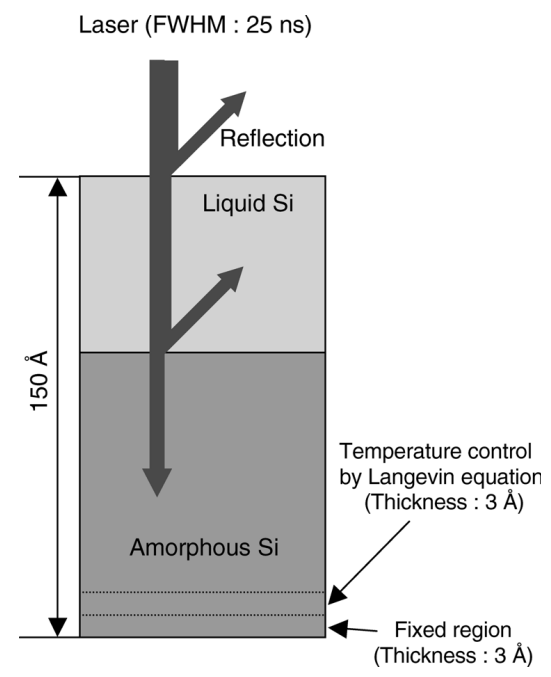

Fig. 3. Schematic diagram of the two-layer model to calculate the deposited laser energy. Absorption in amorphous $\mathrm{Si}$ and $l$-Si layers as well as the reflection at the surface and interface is taken accounts.
$25 \mathrm{~ns}$. In order to simulate the excimer laser annealing, twolayers model was applied in this study as shown in Fig. 3. Liquid Silicon layer was identified by the diffusion coefficient of larger than $8 \times 10^{-7} \mathrm{~cm}^{2} \mathrm{~s}^{-1}$. The refractive indices of amorphous $\mathrm{Si}$ and $l$-Si were set at $n+i k=1.0+3.0 i$ and $n+i k=1.8+3.0 i$, respectively. ${ }^{10)}$ The absorption coefficients of liquid and amorphous $\mathrm{Si}$ were set to same value, $1.5 \times 10^{6} \mathrm{~cm}^{-1}$. The supplied laser energies of atoms were determined by the two-layers model taking into consideration reflections (liquid/amorphous interface and top surface), thickness of layers (liquid and amorphous $\mathrm{Si}$ ) and the distance from surface. The velocities of atoms were scaled depending on the supplied energy. The region with $Z$-coordinates from bottom 3 to $6 \AA$ was controlled to $300 \mathrm{~K}$ by the Langevin equation with the friction constant $\gamma$ set at $2.6 \times 10^{10} \mathrm{~s}^{-1}$.

\section{Results and Discussion}

Figure 4 shows the temperature profile during the irradiation of $180 \mathrm{~mJ} / \mathrm{cm}^{2}$ excimer laser pulse shot. As the irradiation time increases, the temperature of Si films rises and a steady state with a temperature gradient in the direction of the surface normal was obtained within $20 \mathrm{~ns}$. The melting caused from the top surface by heating of the laser energy up to the melting point of amorphous Silicon, $\sim 2000 \mathrm{~K}$. Figure 5 shows melting depth profile with the laser fluence of $180 \mathrm{~mJ} / \mathrm{cm}^{2}$. The melting rate is about $3 \mathrm{~m} / \mathrm{s}$, which values are in good agreement with the experimental data about melting rate during irradiating excimer laser. ${ }^{1)}$ The $180 \mathrm{~mJ} / \mathrm{cm}^{2}$ laser fluence cause complete-melting condition because the excimer laser pulse melts the whole amorphous

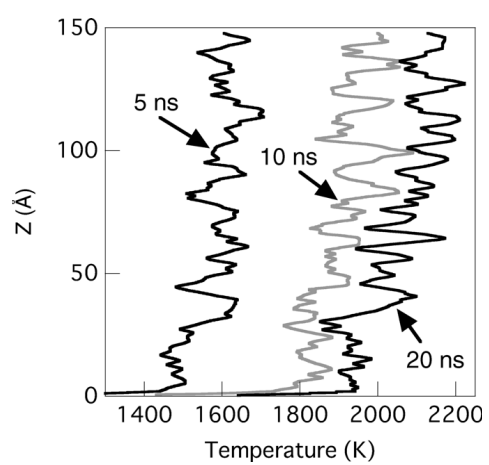

Fig. 4. Temperature profiles along the $Z$-coordinates during the laser of fluence $180 \mathrm{~mJ} / \mathrm{cm}^{2}$.

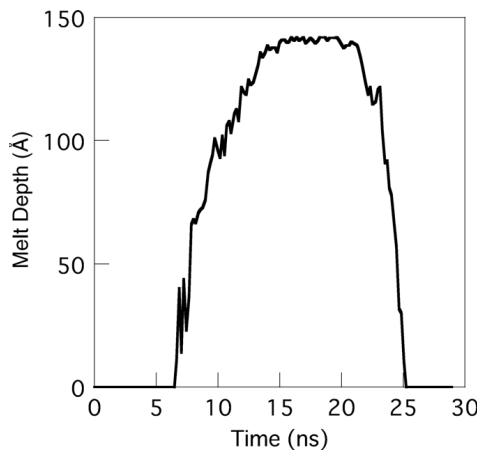

Fig. 5. Melting depth profile during the laser irradiation of $180 \mathrm{~mJ} / \mathrm{cm}^{2}$ fluence. 


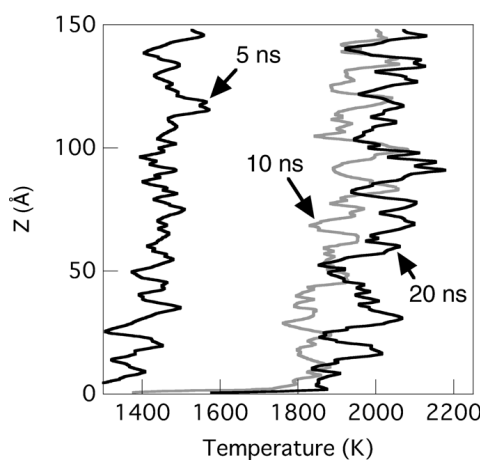

Fig. 6. Temperature profiles along the $Z$-coordinates during the laser of fluence $160 \mathrm{~mJ} / \mathrm{cm}^{2}$.

$\mathrm{Si}$ without the bottom fixed layer. Figure 6 shows the temperature profile during irradiation of $160 \mathrm{~mJ} / \mathrm{cm}^{2}$ excimer laser pulse shot. The melting caused from the top surface by heating of the laser energy up to the melting point of amorphous silicon and a steady state with temperature gradient was obtained after $20 \mathrm{~ns}$ as well as in the case of high fluence laser. However, the temperature of MD cell was wholly low. Figure 7 shows melting depth profile with the values of laser fluence, which are $160 \mathrm{~mJ} / \mathrm{cm}^{2}$. The case of laser fluence $160 \mathrm{~mJ} / \mathrm{cm}^{2}$ was near-complete-melting condition because both $l-\mathrm{Si}$ and amorphous Si phases existed. It was previously reported that the large grains were obtained under the near-complete-melting condition while the spontaneous small grains in amorphous Si region were obtained under the complete-melting condition. ${ }^{1}$ Figure 8 shows diffusion coefficient and temperature profiles at $15 \mathrm{~ns}$ with excimer laser irradiated of fluence $160 \mathrm{~mJ} / \mathrm{cm}^{2}$. The diffusion coefficient $D$ can be given by Einstein equation as follows

$$
D=\lim _{\Delta t \rightarrow \infty} \frac{1}{6 t}\left\langle|r(t+\Delta t)-r(t)|^{2}\right\rangle
$$

where $r(t)$ is the position vector of an atom at time $t$ and $\Delta t$ is the measurement time. In this study, $\Delta t$ was set at $0.1 \mathrm{~ns}$. The temperature of $Z>60 \AA$ is lower than melting point of Tersoff potential while diffusion coefficient is higher than the value of bulk $l$-Si. Therefore it seems that the region of $Z>60 \AA$ is the supercooled liquid. Growth rate of liquid phase epitaxy (LPE) is higher than that of solid phase epitaxy. ${ }^{3,11)}$ Figure 9 shows the snapshots of atomic configuration during excimer laser irradiation with fluence $180 \mathrm{~mJ} /$ $\mathrm{cm}^{2}$. The amorphous $\mathrm{Si}$ was melted from the upper surface region by the laser irradiation as shown in Figs. 9(b) and 9 (c). The $l$-Si region was subsequently quenched and transformed to amorphous Si. After quenching the amorphous $\mathrm{Si}$, spontaneous nucleation was occurred in the amorphous $\mathrm{Si}$ as shown in Figs. 9(e) and 9(f). Figure 10 shows the snapshots of atomic configuration during excimer laser irradiation with fluence $160 \mathrm{~mJ} / \mathrm{cm}^{2}$. The spontaneous nucleation was occurred in unmelted amorphous Si region during melting amorphous $\mathrm{Si}$ from upper region as shown in Fig. 10(c). Figure 10(d) shows that liquid phase reached the nuclei and crystal growth was occurred. After that, explosive crystal growth toward $l$-Si phase was occurred as shown in Figs. 10(e) and 10(f). These results agreed with our prediction that the nucleation occurs from unmelted

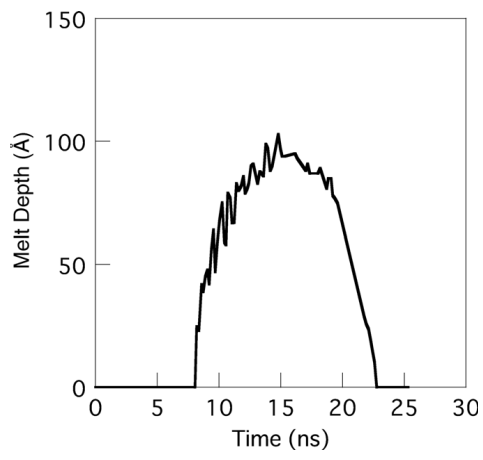

Fig. 7. Melting depth profile during the laser irradiation of $160 \mathrm{~mJ} / \mathrm{cm}^{2}$ fluence.

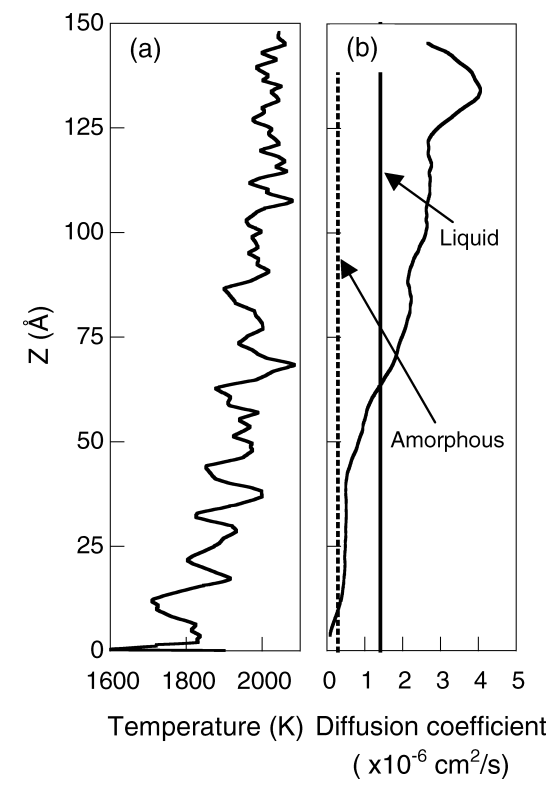

Fig. 8. Temperature (a) and diffusion coefficient (b) profiles along the $Z$-coordinates at $15 \mathrm{~ns}$ for the laser with fluence of $160 \mathrm{~mJ} / \mathrm{cm}^{2}$. The solid and broken lines in (b) show the diffusion coefficients of bulk $l$-Si and amorphous Si obtained by our simulations, respectively.

amorphous Si region during laser irradiation and crystal growth proceeds toward supercooled $l$-Si region in the nearcomplete-melting condition. ${ }^{2)}$

\section{Conclusions}

We have directly observed the crystallization process of excimer laser annealing of amorphous Si thin films using MD simulations in consideration of reflection and absorption. The temperature and diffusion coefficient profiles of Si film were investigated during excimer laser annealing. A steady state temperature gradient was obtained in the direction of the surface normal during laser irradiation. In the complete-melting condition, the melting of amorphous $\mathrm{Si}$ occurred by laser irradiation and subsequently quenched $l$ $\mathrm{Si}$ was transformed to amorphous Si. Nucleation was occurred in the quenched amorphous $\mathrm{Si}$ and grain size is small in complete-melting condition. On the other hand in near-complete-melting condition, nucleation was occurred in the unmelted amorphous Si region during laser irradiation. The grain size was enlarged in the near-complete- 


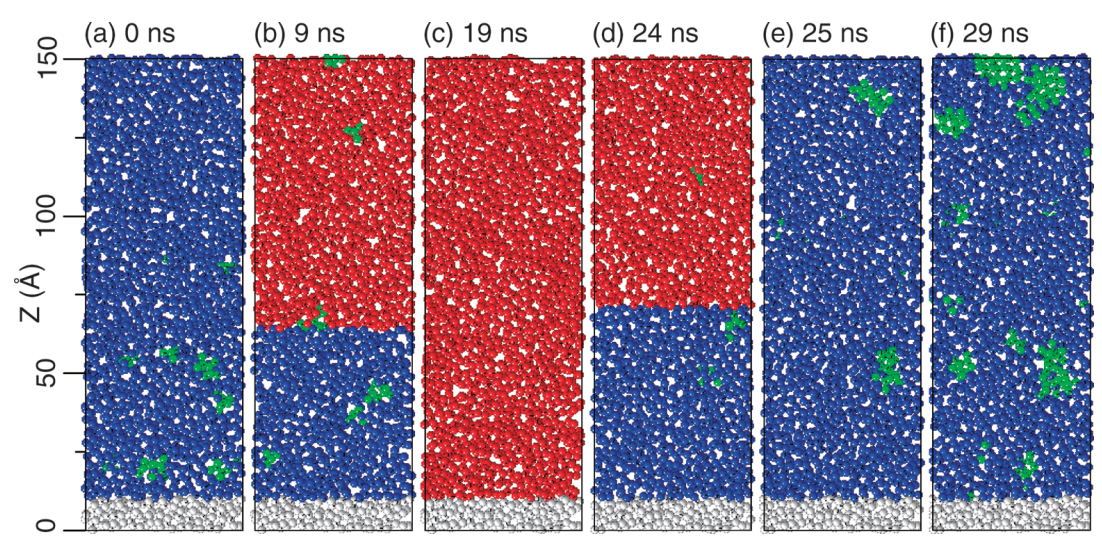

Fig. 9. Snapshots of atomic configurations during the laser irradiation with the fluence of $180 \mathrm{~mJ} / \mathrm{cm}^{2}$ : (a) initial, after (b) $9 \mathrm{~ns}$, (c) $19 \mathrm{~ns}$, (d) $24 \mathrm{~ns}$, (e) $25 \mathrm{~ns}$ and (f) $29 \mathrm{~ns}$. Amorphous Si atoms are shown in blue, liquid Si atoms are shown in red, well-ordered atoms are shown in green and the fixed region is shown in white. These snapshots were obtained by projecting all the atoms in the MD cells in the $5 \AA$ thick region from 45 to $50 \AA$ in the $\mathrm{X}$ direction.

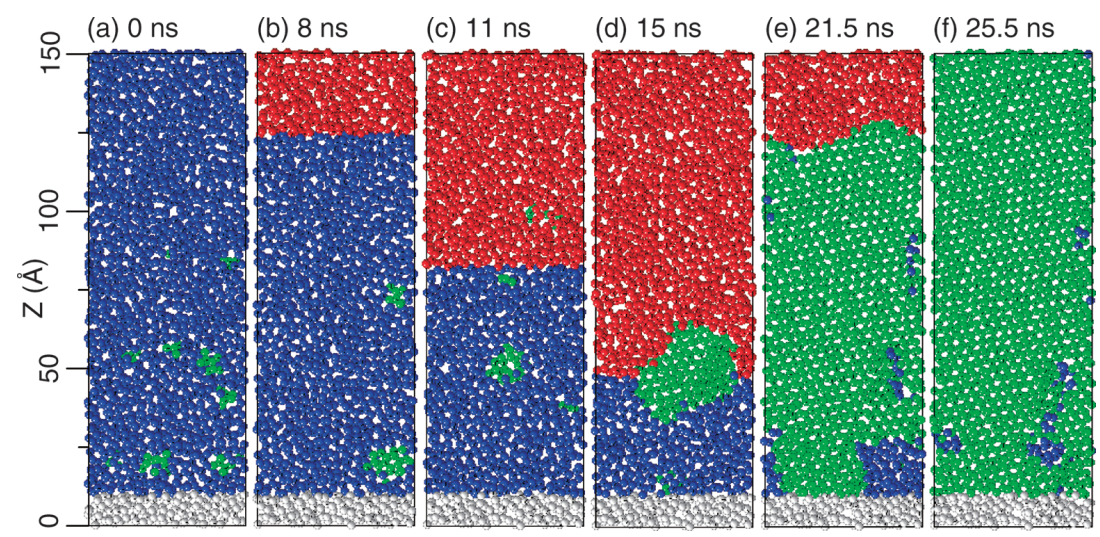

Fig. 10. Snapshots of atomic configurations during the laser irradiation with the fluence of $160 \mathrm{~mJ} / \mathrm{cm}^{2}$ : (a) initial, after (b) $8 \mathrm{~ns}$, (c) $11 \mathrm{~ns}$, (d) $15 \mathrm{~ns}$, (e) $21.5 \mathrm{~ns}$ and (f) $25.5 \mathrm{~ns}$. Amorphous Si atoms are shown in blue, liquid Si atoms are shown in red, well-ordered atoms are shown in green and the fixed region is shown in white. These snapshots were obtained by projecting all the atoms in the MD cells in the $5 \AA$ thick region from 45 to $50 \AA$ in the $\mathrm{X}$ direction.

melting condition because crystal growth toward supercooled liquid Si.

\section{REFERENCES}

1) M. Hatano, S. Moon, M. Lee, K. Suzuki and C. P. Grigoropoulos: $J$. Appl. Phys., 87 (2000), 36.

2) S. Munetoh, T. Kuranaga, B. M. Lee, T. Motooka, T. Endo and T. Warabisako: Jpn. J. Appl. Phys., 45 (2006), 4344.

3) S. Munetoh, T. Kuranaga, B. M. Lee, T. Motooka, T. Endo and T. Warabisako: Dig. Tech. Papers AM-LCD'05, (2005), 295.

4) S. Munetoh, T. Mitani, T. Kuranaga and T. Motooka: MRS Proc., 0958 (2006), L07-07.
5) J. Tersoff: Phys. Rev. B, 38 (1988), 9902.

6) J. Tersoff: Phys. Rev. B, 39 (1989), 5566.

7) S. J. Cook and P. Clancy: Phys. Rev. B, 47 (1993), 7686

8) S. C. Moss and J. F. Graczyk: Proc. of the 10th Int. Conf. on the Physics of Semicondoctors, U.S. Atomic Energy Commission, Oak Ridge, (1970), 658.

9) W. A. Kamitakahara, H. R. Shank, J. F. McClelland, U. Buchenau, F. Gompf and L. Pintschovins: Phys. Rev. Lett., 52 (1984), 644.

10) M. Serenyi, T. Lohner, P. Petrik and C. Frigeri: Thin Solid Films, 515 (2007), 3559.

11) T. Motooka, K. Nisihira, S. Munetoh, K. Moriguchi and A. Shintani: Phys. Rev. B, 61 (2000), 8537. 